\title{
Ranolazine, But not Trimetazidine, Influences Markers of Inflammation in Patients with Stable Coronary Artery Disease
}

\author{
Andreja Rehberger Likozar ${ }^{1}$ and Miran Šebeštjen ${ }^{1-3 *}$ \\ ${ }^{1}$ Department of Vascular Diseases, University Medical Centre Ljubljana, Slovenia \\ ${ }^{2}$ Department of Cardiology, University Medical Centre Ljubljana, Slovenia \\ ${ }^{3}$ Faculty of Medicine, University of Ljubljana, Slovenia
}

*Corresponding author: Miran Šebeštjen, Department of Cardiology University Medical Centre Ljubljana Zaloška 7, 1000 Ljubljana, Slovenia

\section{ARTICLE INFO}

Received: 彗 March 18, 2021

Published: 㗀 March 24, 2021

Citation: Andreja Rehberger Likozar, Miran Šebeštjen. Ranolazine, But not Trimetazidine, Influences Markers of Inflammation in Patients with Stable Coronary Artery Disease. Biomed J Sci \& Tech Res 34(4)-2021. BJSTR. MS.ID.005598.

Keywords: Coronary Artery Disease; Inflammatory Parameters; Ranolazine; Trimetazidine; C-Reactive Protein; Interleukin-10

Abbreviations: CAD: Coronary Artery Disease, CRP: C-Reactive Protein, ACE: Angiotensin-Converting Enzyme, ARBs: Angiotensin Receptor Blockers, VCAM: Vascular Adhesion Molecule

\section{ABSTRACT}

Purpose: Ranolazine and trimetazidine have been individually shown to reduce the levels of inflammatory parameters in patients with coronary artery disease (CAD). However, no direct comparison between these two drugs has been made in these patients. The aim was to compare the effects in patients with stable CAD of ranolazine and trimetazidine on C-reactive protein (CRP), tumor necrosis factor- $\alpha$ (TNF- $\alpha$ ), interleukin (IL)-6 and IL-10, and vascular adhesion molecule (VCAM) 1.

Methods: We randomised 56 patients with stable CAD to 12 weeks treatment with trimetazidine $35 \mathrm{mg}$ twice daily, or with ranolazine $375 \mathrm{mg}$ twice daily for 4 weeks, then increased to ranolazine $500 \mathrm{mg}$ twice daily.

Results: Ranolazine significantly decreased CRP from 0.97 (0.45-7.80) to 0.75 (0.22$1.95) \mathrm{mg} / \mathrm{L}(\mathrm{p}=0.03)$, with no changes with trimetazidine, as $0.79(0.64-5.00)$ to 0.89 $(0.43-3.00) \mathrm{mg} / \mathrm{L}(\mathrm{p}=0.95)$. Ranolazine lowered IL-10 from $1.20(0.90-1.50)$ to 0.80 $(0.70-1.10) \mathrm{ng} / \mathrm{L}(\mathrm{p}=0.01)$, with no changes for trimetazidine, as $1.30(1.10-1.90)$ to 1.30 $(1.00-1.70) \mathrm{ng} / \mathrm{L}(\mathrm{p}=0.88)$. No significant changes were seen for TNF- $\alpha$, IL-6 and VCAM1 in either treatment group.

Conclusion: This study shows that for patients with CAD, ranolazine significantly lowers two markers of inflammation, CRP and IL-10, while trimetazidine has no influence on any of the examined inflammation markers. These data suggest further beneficial effects on some inflammatory markers in patients with CAD that can be attributed to ranolazine actions, but not for trimetazidine. The reduction of these inflammatory markers might explain the basis for the antianginal effects of ranolazine in patients with CAD.

\section{Introduction}

Inflammation has a critical role in the initiation and progression of the atherosclerotic process [1], with both pro-inflammatory and anti-inflammatory cytokines involved. The fate of atherosclerotic plaques and the clinical complications that can arise result from the balance between the pro-inflammatory and anti- inflammatory mediators that regulate the magnitude of the inflammatory response within plaques [2,3]. Among the inflammatory parameters,
C-reactive protein (CRP) has been shown to be the most important factor in the prediction of future coronary artery events [4]. CRP is mainly synthesised in the liver, although extrahepatic transcription of CRP upon inflammatory stimuli with interleukin (IL)-1, IL-6 and tumor necrosis factor (TNF)- $\alpha$ has been described $[5,6]$. TNF- $\alpha$ is a multifunctional circulating cytokine that is the principal mediator of the acute inflammatory response. 
It is derived from endothelial cells and macrophages associated with coronary atheroma. TNF- $\alpha$ has been shown to be elevated in patients with previous myocardial infarction with increased risk of recurrent coronary events [7]. Together with the cytokines and IL-6, TNF- $\alpha$ stimulates synthesis of the acute-phase proteins [8], and its levels correlate closely with CRP serum levels [9] IL-10 and transforming growth factor- $\beta$ (TGF- $\beta$ ) are the most prominent antiinflammatory cytokines. Both IL-10 and TGF- $\beta$ can protect against atherogenesis and inhibit macrophage and lymphocyte function. They can also stimulate the generation of regulatory $\mathrm{T}$ cells, which can in turn promote more IL-10- and TGF- $\beta$-producing cells [10]. Despite the biological plausibility that IL-10 is a cardioprotective cytokine, elevated baseline plasma levels of IL-10 were shown to be a strong and independent predictor of long-term adverse cardiovascular outcomes in patients with acute coronary syndrome [11]. Increased expression of adhesion molecules by the activated endothelium is a critical feature of atherosclerosis. In patients with acute coronary syndromes, increased serum levels of vascular adhesion molecule (VCAM) 1 powerfully predict increased risk for subsequent cardiovascular events [12].

Ranolazine reduces intracellular sodium and calcium concentrations in ischaemic myocytes through inhibition of the late phase of the inward sodium current, and in this way it decreases the related detrimental effects of ischaemia $[13,14]$. It has been shown that ranolazine decreases CRP levels in patients with stable angina pectoris, although no possible mechanism for this effect has been described [15]. Trimetazidine inhibits long-chain 3-ketoacyl-CoAthiolase and shifts the myocardial metabolism from fatty acids to the oxidation of glucose. As a result, myocardial energy metabolism is improved and intracellular acidosis and calcium deposition are decreased [16]. Trimetazidine protects tissues and limits cell membrane damage from free radicals, which can be associated with the release of some pro-inflammatory mediators [17].

In the latest guidelines, both ranolazine and trimetazidine are recommended as second-line treatments in the management of chronic stable angina pectoris, and although they do not influence patient morbidity and mortality, they improve patient quality of life [18]. However, there is no direct comparison of the effects on the levels of inflammatory parameters of each of these drugs. Therefore, the aim of the present study was to compare the effects of ranolazine and trimetazidine on the above-mentioned inflammatory parameters in patients with stable coronary artery disease (CAD).

\section{Material and Methods}

\section{Study Design}

This was prospective randomised, double-blind study. After initial screening, 53 men who satisfied the inclusion criteria were exposed to 12 weeks of treatment with either ranolazine or trimetazidine. Ranolazine was given at a dose of $375 \mathrm{mg}$ twice daily for 4 weeks, with an increase to ranolazine $500 \mathrm{mg}$ twice daily for the following 8 weeks. Trimetazidine was given as a dose of $35 \mathrm{mg}$ twice daily throughout the study. To retain the doubleblind nature of the study, despite the increase in the dose in the ranolazine group, both groups of patients received their medication in two different boxes. The first box contained the medication for the first 4 weeks, and the second box for the following 8 weeks, to the conclusion of the study. The randomization was performed using the Research Randomizer computer software, version 4.0. Before randomization and after 12 weeks of treatment, clinical examinations, electrocardiograms and laboratory blood analyses were performed. G*power 3.1 programme [19] was used to calculate sample size given $80 \%$ power of the study and 0.05 a error probability.

\section{Patients}

Male patients with clinically stable CAD of at least 12 months after myocardial infarction who were aged between 18 and 65 years were eligible for participation in the study. All of the patients had previously already received $\beta$-blockers and antiplatelet drugs, angiotensin-converting enzyme (ACE) inhibitors/ angiotensin receptor blockers (ARBs) and statins. Before entering the study, the therapies of all of the patients were left unchanged for at least 6 months. Patients with hypersensitivity to ranolazine or trimetazidine, elevated liver transaminases $(>3$ times normal values), severe renal impairment and serum creatinine levels $>200 \mathrm{mmol} / \mathrm{L}$, prolonged QT interval, history of acute illness in the previous 6 weeks, or previous use of ranolazine or trimetazidine were eliminated from the study group. All of the procedures performed in this study that involved human participants were carried out in accordance with the ethical guidelines of the 1964 Declaration of Helsinki. Approval for this study was obtained from the National Medical Ethics Committee of the Republic of Slovenia (reference number: KME 127/10/13). Written informed consent was obtained from all of the patients included in this study.

\section{Clinical Examination}

During the clinical examinations of the patients, their blood pressures were measured. Their anthropometric parameters were determined, and their body mass index (BMI) was calculated.

\section{Laboratory Procedures}

Blood samples for laboratory analysis were collected in the morning after a 12-h fasting period. The serum concentrations of total cholesterol, high-density lipoprotein (HDL)-cholesterol, and triglycerides were determined using standard colorimetric assays (Fusion FS 5.1 Analyzer: Ortho-Clinical Diagnostics, USA). Friedewald's formula was used to calculate low-density lipoprotein (LDL)- [20]. Serum CRP was measured using a standard colorimetric assay with the CRP microslide test on a chemical analyzer (Vitros Fusion 5.1; Ortho-Clinical Diagnostics, USA). 
Values below $10 \mathrm{mg} / \mathrm{L}$ were measured again with hsCRP MicroTip tests on the same analyser. Enzyme-linked immunosorbent assays (ELISAs) were used to measure the serum levels of IL-6 (Quantikine(r) HS Human IL-6 Immunoassay), IL-10 (Quantikine(r) HS Human IL-10 Immunoassay), TNF- $\alpha$ (Quantikine(r) HS Human TNF-a Immunoassay) and VCAM1 (Quantikine(r) Human VCAM1 Immunoassay), according to the instructions of the manufacturer (R\&D Systems, USA).

\section{Statistical Analysis}

Kolmogorov-Smirnov tests were used to define variables showing normal distributions, with these data expressed as means \pm standard deviations. The non-normally distributed variables are expressed as medians and range (lower and upper quartile). Baseline differences between the study groups were tested for significance using Student's t-tests for unpaired data for normally distributed variables and using Mann- Whitney U-tests for nonnormally distributed variables. The changes between baseline and after 12 weeks of treatment presented in Figures 1 \& 2 were calculated from individual patient data using the following equation: (value at week 12-value at baseline)/value at baseline and expressed as percentage. The changes in each variable after the intervention period in each group were compared using Wilcoxon signed-rank tests. Post-hoc comparisons were carried out using Tukey HSD tests. The criterion for statistical significance was $p$ $<0.05$. All of the calculations were performed using the Statistica programme (StatSoft Inc.7.0., USA) and graphs were drawn using GraphPad Prism, version 6.07 for Windows (GraphPad Software, La Jolla, CA, USA; www.graphpad.com).

\section{Result}

\section{Patient Characteristics}

The study population comprised 53 male patients (mean age, 57 years) with optimally treated and stable CAD. These patients were randomized to either the ranolazine treatment group $(n=27$; mean age, 58 years) or the trimetazidine treatment group $(n=26$; mean age, 56 years). From at least 6 months prior to inclusion in the study and throughout the study, the patient background therapies were not changed. In the ranolazine group, three patients did not complete the study due to adverse events (one arrhythmia, one chest pain, one fatigue and muscle pain), and one patient withdrew for personal reasons. In the trimetazidine group, three patients did not complete the study for personal reasons. The clinical characteristics of the final study group are shown in Table 1. All of the clinical characteristics remained unchanged from baseline to the end of the treatment period.

In the ranolazine group, 18 patients had single vessel disease, two had two-vessel disease, and three had multi-vessel disease. In the trimetazidine group, there were 17 patients with single vessel disease, five with two-vessel disease, and one with multi-vessel disease. In the ranolazine group, 20 patients had been treated with percutaneous revascularization at the time of an acute event, while three had needed surgical revascularization. In the trimetazidine group, 21 patients had been treated with percutaneous revascularization, and two had needed surgical revascularization. In the ranolazine group, 20 patients were receiving bisoprolol (mean dose, $7.8 \mathrm{mg}$ daily) and three patients were receiving carvedilol (mean dose, $33 \mathrm{mg}$ daily). In the trimetazidine group, all of the patients were receiving bisoprolol (mean dose, $8.1 \mathrm{mg}$ daily). In both groups, two patients were receiving ARBs (losartan, candesartan), while all of the other patients were receiving various ACE inhibitors (perindopril, ramipril, trandolapril, enalapril). Only one patient in each group was receiving a calcium channel blocker (amlodipine $10 \mathrm{mg}$ daily). All of the patients were under treatments with statins at doses designed to maintain LDL-cholesterol levels $<1.8 \mathrm{mmol} / \mathrm{L}$. All of the patients also received acetylsalicylic acid $100 \mathrm{mg}$ daily. All of the patients had normal left ventricular ejection fraction $>55 \%$.

\section{Biochemical Parameters}

The data for the biochemical parameters are presented in Table 2 \& Figure 1. There were no differences in the serum lipids and blood glucose between the two treatment groups at baseline. After 12 weeks of treatment, the only significant change seen was for the increase in triglycerides from baseline in the trimetazidine group; however, compared with the ranolazine data, significance was not reached for either the difference in the triglyceride changes or the triglyceride levels at the end of the study. In both of the treatment groups, there were no significant changes in blood glucose, total cholesterol, LDL-cholesterol or HDL-cholesterol (Table 2 \& Figure 1).

Table 1: Mean baseline clinical characteristics of the patients included in this study.

\begin{tabular}{|c|c|c|c|c|}
\hline Parameter & Units & Ranolazine (n = 23) & Trimetazidine $(n=23)$ & $\mathbf{p}$ \\
\hline Age & years & $57.9 \pm 6.7$ & $56.1 \pm 6.2$ & 0.303 \\
\hline Body mass index & $\mathrm{kg} / \mathrm{m} 2$ & $30.5 \pm 4.5$ & $29.2 \pm 4.1$ & 0.283 \\
\hline Systolic blood pressure & $\mathrm{mmHg}$ & $115 \pm 16$ & $119 \pm 13$ & 0.334 \\
\hline Diastolic blood pressure & $\mathrm{mmHg}$ & $68 \pm 10$ & $68 \pm 11$ & 0.977 \\
\hline Heart rate & beats/min & $63 \pm 12$ & $61 \pm 10$ & 0.426 \\
\hline QTc interval & $\mathrm{ms}$ & $393 \pm 23$ & $404 \pm 21$ & 0.099 \\
\hline
\end{tabular}


Table 2: Biochemical parameters at baseline and after 12 weeks of treatments with ranolazine or trimetazidine.

\begin{tabular}{|c|c|c|c|c|}
\hline Parameter (mmol/L) & Treatment Group & Baseline & After 12 Weeks & $\mathbf{p}$ \\
\hline \multirow{2}{*}{ Blood glucose } & Ranolazine & $6.13 \pm 1.19$ & $6.29 \pm 1.41$ & 0.63 \\
\hline & Trimetazidine & $6.77 \pm 2.74$ & $6.36 \pm 2.23$ & 0.25 \\
\hline \multirow{2}{*}{ Total cholesterol } & Ranolazine & $3.93 \pm 0.98$ & $3.93 \pm 0.83$ & 0.99 \\
\hline & Trimetazidine & $3.77 \pm 0.82$ & $3.77 \pm 0.96$ & 0.99 \\
\hline \multirow{2}{*}{ LDL cholesterol } & Ranolazine & $1.91 \pm 0.73$ & $1.89 \pm 0.63$ & 0.83 \\
\hline & Trimetazidine & $1.76 \pm 0.60$ & $1.79 \pm 0.61$ & 0.79 \\
\hline \multirow{2}{*}{ HDL cholesterol } & Ranolazine & $1.20 \pm 0.37$ & $1.20 \pm 0.30$ & 0.86 \\
\hline & Trimetazidine & $1.09 \pm 0.27$ & $1.05 \pm 0.21$ & 0.24 \\
\hline \multirow{2}{*}{ Triglycerides } & Ranolazine & $1.45(1.05-2.01)$ & $1.54(1.20-2.05)$ & 0.52 \\
\hline & Trimetazidine & $1.36(1.04-2.13)$ & $1.42(1.12-2.58)$ & $0.04^{*}$ \\
\hline
\end{tabular}

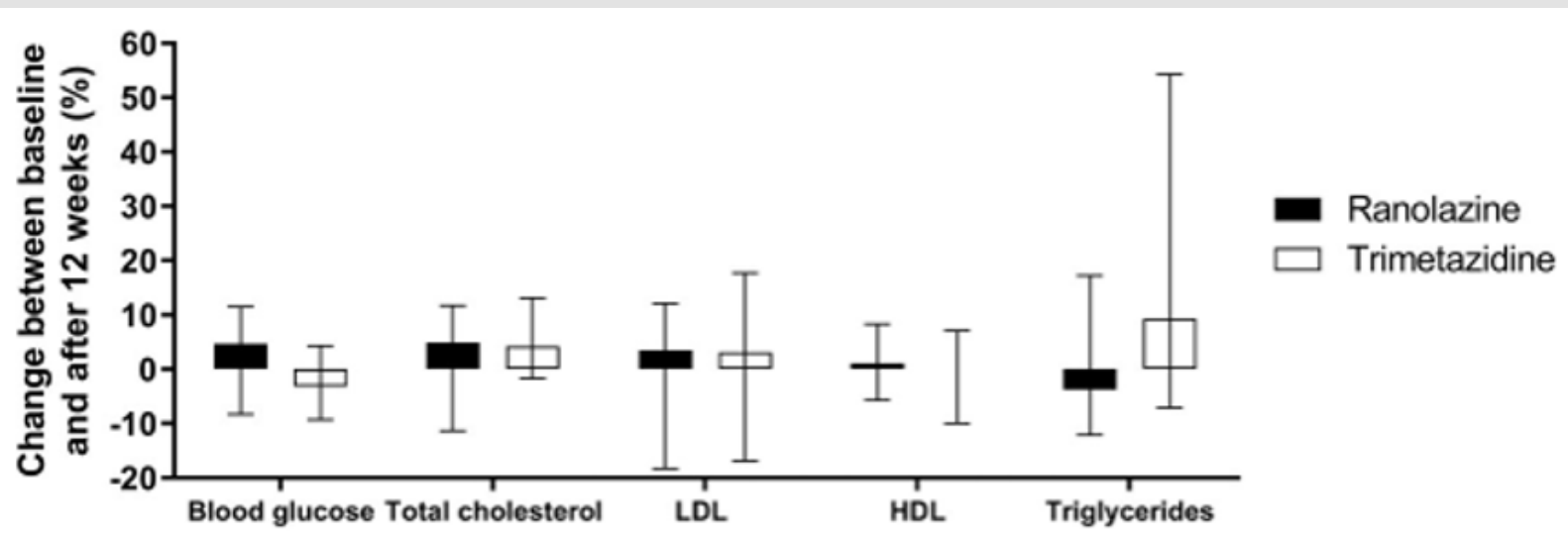

Figure 1: Changes in biochemical parameters following ranolazine and trimetazidine treatments.

Note: Changes from baseline to 12 weeks of treatment with ranolazine and trimetazidine treatments, expressed as percentages of baseline values. Data are medians and range (lower and upper quartile). ${ }^{*}, p<0.05$.

\section{Markers of Inflammation}

The data for the markers of inflammation examined here are presented in Table 3 \& Figure 2. At baseline, there were no significant differences between the two treatment groups for the serum levels of CRP, TNF- $\alpha$, IL- 6, IL-10 or VCAM1. However, a statistically significant decrease in serum CRP levels was observed in the ranolazine group, but not in the trimetazidine group. In the ranolazine group, serum CRP values dropped by $23.2 \%(\mathrm{p}=0.03)$, whereas in the trimetazidine group there was no statistical significance $(\mathrm{p}=0.95)$. These changes in CRP defined a significant difference between the two treatment groups $(\mathrm{p}=0.045)$ (Figure 2). IL-10 decreased significantly in the ranolazine group $(25.4 \%$; $\mathrm{p}=0.01$ ), while in the trimetazidine group the IL-10 levels were not changed from before to after the treatment $(p=0.88)$ (Table 3). These changes in IL-10 defined a further significant difference between the two treatment groups $(p=0.034)$ (Figure 2). No significant changes were observed in the serum levels of TNF- $\alpha$, IL-6 and VCAM1 from baseline to 12 weeks for either of the treatment groups (Table 3). Similarly, there were no significant differences in the changes in the TNF- $\alpha$, IL- 6 and VCAM1 inflammatory markers between the two treatments (Figure 2).

Table 3: Inflammatory parameters at baseline and after 12 weeks of treatment with ranolazine or trimetazidine.

\begin{tabular}{|c|c|c|c|c|c|}
\hline Parameter & Units & Treatment Group & Baseline & After 12 weeks & p \\
\hline \multirow{2}{*}{ CRP } & \multirow{2}{*}{$\mathrm{mg} / \mathrm{L}$} & Ranolazine & $0.97(0.45-7.80)$ & $0.75(0.22-1.95)$ & $0.03^{*}$ \\
\hline & & Trimetazidine & $0.79(0.64-5.00)$ & $0.89(0.43-3.00)$ & 0.95 \\
\hline \multirow{2}{*}{ TNF- $\alpha$} & \multirow{2}{*}{$\mathrm{ng} / \mathrm{L}$} & Ranolazine & $5.60(4.20-14.60)$ & $3.50(2.20-15.70)$ & 0.25 \\
\hline & & Trimetazidine & $12.10(3.50-37.20)$ & $9.90(3.20-21.30)$ & 0.61 \\
\hline \multirow{2}{*}{ IL-6 } & \multirow{2}{*}{$\mathrm{ng} / \mathrm{L}$} & Ranolazine & $2.80(2.10-4.00)$ & $2.50(1.80-3.20)$ & 0.17 \\
\hline & & Trimetazidine & $2.60(2.20-4.00)$ & $2.60(2.10-4.70)$ & 0.75 \\
\hline
\end{tabular}




\begin{tabular}{|c|c|c|c|c|c|}
\hline \multirow{2}{*}{ IL-10 } & \multirow{2}{*}{$\mathrm{ng} / \mathrm{L}$} & Ranolazine & $1.20(0.90-1.50)$ & $0.80(0.70-1.10)$ & $0.01^{*}$ \\
\cline { 3 - 6 } & & Trimetazidine & $1.30(1.10-1.90)$ & $1.30(1.00-1.70)$ & 0.88 \\
\hline \multirow{2}{*}{ VCAM1 } & \multirow{2}{*}{$\mu \mathrm{g} / \mathrm{L}$} & Ranolazine & $884(726-977)$ & $797(700-977)$ & 0.61 \\
\cline { 3 - 7 } & & Trimetazidine & $916(787-1189)$ & $922(767-1259)$ & 0.98 \\
\hline
\end{tabular}

Note: Data are as medians (lower quartile-upper quartile). P values are for the difference between baseline and 12 weeks for each treatment group; CRP, C-reactive protein; IL, interleukin; TNF- $\alpha$, tumor necrosis factor $a$, VCAM1, vascular cell adhesion molecule 1. * $\mathrm{p} \leq 0.05$.

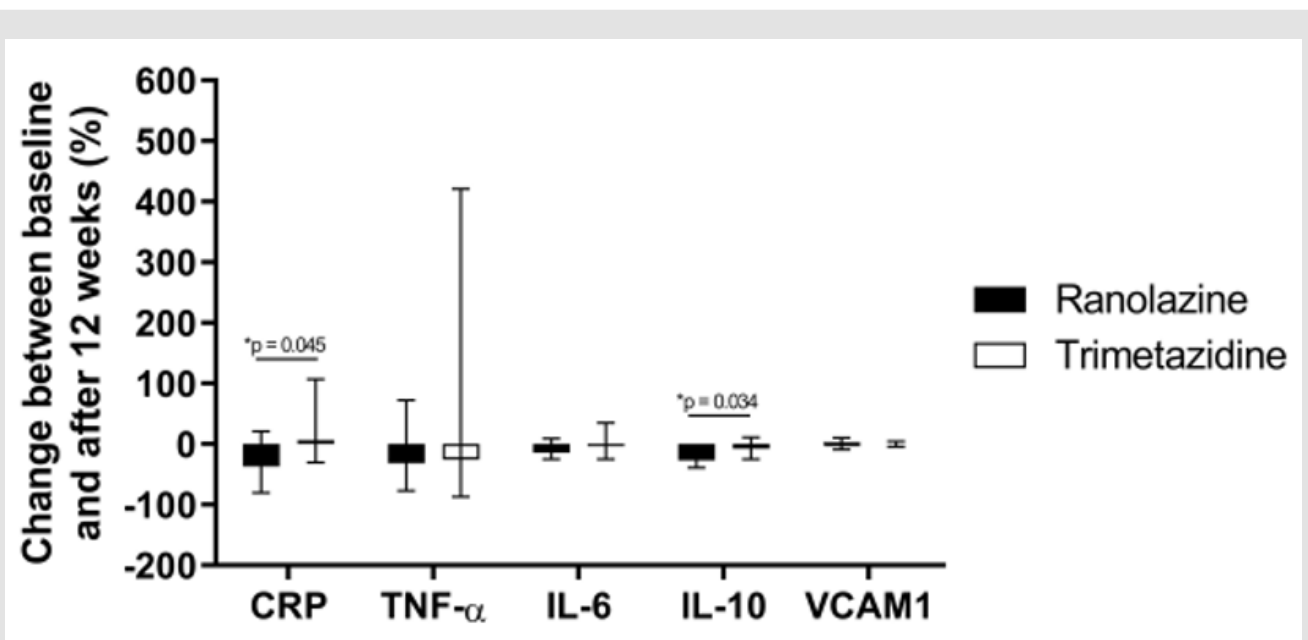

Figure 2: Changes in inflammatory markers following ranolazine and trimetazidine treatments.

Note: Changes from baseline to 12 weeks of treatment with ranolazine and trimetazidine treatments, expressed as percentages of baseline values. Data are medians and range (lower and upper quartile). ${ }^{*}, \mathrm{p}<0.05$. CRP, C- reactive protein; IL-6, interleukin 6; IL-10, interleukin 10; TNF-a, tumor necrosis factor a; VCAM1, vascular cell adhesion molecule 1.

\section{Discussion}

Although ranolazine and trimetazidine are second-line drugs for treatment of stable CAD, it is important to evaluate their effects on inflammation, as this process is a risk factor for acute coronary events. Therefore, we performed this first prospective, randomized trial that compared the effects of ranolazine and trimetazidine on markers of inflammation in optimally treated patients with stable CAD. Here, the serum CRP levels significantly decreased only in the ranolazine group, while there was no significant change in the trimetazidine group. The only study that has investigated the influence of ranolazine on CRP serum levels in patients with stable CAD was by Deshmukh, et al. [15], who reported that ranolazine decreased serum CRP levels in comparison with placebo. The present study showed similar effects on CRP levels, although here the difference was that we compared ranolazine to trimetazidine. In both of these studies, all of the patients were already under treatment with statins, which have been shown to decrease serum CRP levels [21-24]. One particular difference between their study [15] and the present study was that all of the patients here were also treated either with ACE inhibitors or sartans; in the Deshmukh et al. study, only three- quarters of the patients received ACE inhibitors or sartans [15]. However, it is unlikely that this is of any importance.
The mechanism by which ranolazine decreases CRP is unknown. We found no changes in the levels of IL-6, which is the central mediator of the acute-phase response and a primary stimulator of hepatic production of CRP [25]. It appears that the decrease in CRP after the treatment with ranolazine was not dependent on decreased CRP production in the liver, where IL-6 would be the primary trigger. At first sight, these data might look surprising, but it has been shown that patients with stable angina pectoris have comparable levels of CRP and IL-6 to the healthy population [26], with no correlation between these two parameters in either of these groups. On the other hand, for patients with acute myocardial infarction and unstable angina pectoris, it has been shown that the levels of both CRP and IL-6 were significantly higher and interdependent [26]. In the present group of patients, there was no correlation between changes in the serum levels of CRP and IL-6. This might be because in the present study all of these patients were treated with ACE [27] or sartans [28], statins [29] and acetylsalicylic acid [30], which are known to decrease the levels of both CRP and IL-6. It is not known whether concomitant treatment with ACE inhibitors or sartans, statins and acetylsalicylic acid, or also ranolazine or trimetazidine, have any influence on CRP levels. Here there were no changes in CRP or IL-6 levels in the trematizidine treatment group. To the best of our knowledge, no such study has been published previously. Indeed, it might be 
that ranolazine and trimetazidine decrease CRP and IL-6 through different mechanisms.

In the present study, there were no changes in serum CRP levels in the patients who were treated with trimetazidine. On the other hand, Pudil, et al. [31] reported that compared to placebo, there was a lower increase in CRP in patients after acute myocardial infarction who were treated with streptokinase and who received trimetazidine. Then, as in the present study, they did not find any change in the levels of IL- 6 with this trimetazidine treatment. We can therefore assume that the inhibition of CRP production in their patients was not suppressed via the IL-6 pathway. In similar patients to those in the present study, Szkodzinski, et al. [32] reported that a 3-month treatment with trimetazidine decreased CRP levels, with no changes in IL-6 levels. We have no data regarding treatments with statins in their study, which might be the reason for the differences in these results.

In patients with acute coronary syndrome, a 3-day pretreatment with trimetazidine before percutaneous intervention did not influence CRP levels [16]. On the contrary, Kuralay et al. (Kuralay, et al. [17]) reported that a 3-day pre-treatment with trimetazidine significantly reduced the increased levels of CRP and TNF- $\alpha$ seen after percutaneous coronary intervention in patients with stable coronary artery disease. Martins, et al. [33] instead looked at patients who were to undergo coronary artery bypass, where they showed that compared to placebo, their treatment with trimetazidine for 12-15 days before grafting had no effects on CRP release during and $48 \mathrm{~h}$ after the procedure. However, they reported a lower increase in IL-6 in patients treated with trimetazidine compared to placebo. The ischaemic and reperfused myocardium has been shown to be the major source of IL- 6 during coronary bypass grafting [34] and percutaneous coronary revascularization [31], with a peak increase that occurs immediately after the procedure, with a peak in CRP levels observed $18 \mathrm{~h}$ later [31]. This might mean that production of CRP is triggered by an increased production of IL-6. However, we cannot compare these studies $[31,33]$ with the present study, as previously they examined the acute effects of trimetazidine on provoked inflammatory responses after percutaneous myocardial revascularization, while in the present study the patients were treated with trimetazidine for 12 weeks; therefore, the inflammatory parameters were significantly lower than in their studies. On the other hand, in patients with ischaemic dilated cardiomyopathy, long-term treatment with trimetazidine produces smaller increases in CRP levels compared with placebo [35]. They did not examine the serum levels of the other cytokines that were measured in the present study, where there were no changes in the levels of VCAM, IL-6 or IL-10 in the patients treated with trimetazidine. We have not found any studies that have examined the effects of trimetazidine on these parameters in patients with CAD or any other clinically evident atherosclerotic disease. We saw here in the present study that IL10 levels decreased after treatment with ranolazine, even though IL-10 has been shown previously to mediate anti-atherogenic pathways. For example, in preclinical models, IL-10 promoted the stability of atherosclerotic plaques [36]. Patients after acute myocardial infarction with elevated IL-10 levels had a decreased risk of death or non-fatal myocardial infarction [37]. Also, it was shown that patients with elevated CRP and IL-10 were at lower risk than those with elevated CRP only. On the other hand, there was no correlation between IL-10 levels and future cardiovascular events in patients with stable CAD $[38,39]$, as also seen in the present study. Higher levels of IL-10 at the time of an acute coronary event might only be the consequence of response to circulating cytokines, such as IL- 6 and TNF- $\alpha$, that act as surrogate markers of an overall pro- inflammatory milieu $[40,41]$.

In a Multi-Ethnic Study of Atherosclerosis (MESA) [42], that included 930 patients with no clinical signs of atherosclerosis, the patient baseline IL-10 levels appeared to be unrelated to cardiovascular events and were a poor marker of subclinical coronary atherosclerosis. Inoue et al. [43] evaluated the serum levels of 10 cytokines as potential markers of long-term outcomes in patients with angiographically identified stable CAD. They reported that independent of the other nine cytokines and high sensitivity CRP, IL-8 can predict cardiovascular events [43]. The main limitation of the present study is the relatively small number of patients. These data should thus be confirmed in larger controlled trials.

\section{Conclusion}

In patients with stable CAD, treatment with ranolazine decreases the levels of serum CRP and the anti- inflammatory cytokine IL10 , changes that are not seen for trimetazidine treatment of these patients. The role of this cytokine as a prognostic marker for acute coronary events is still under debate. Although neither of these drugs has any effects on mortality and morbidity in patients with stable chronic artery disease, ranolazine might affect the plaque rupture via lowering CRP, which should be definitely verified in future studies.

\section{Declarations}

\section{Funding}

This study did not receive any specific grant, funding or other payments, goods or services that might influence the work.

\section{Conflicts of Interest/Competing Interests}

Both authors declare that there is no conflict of interest regarding the publication of this article. Both authors confirm that we accept responsibility for the final manuscript, and that we agree with its content and its form. 


\section{Ethics Approval (Include Appropriate Approvals or Waivers)}

Approval for this study was obtained from the National Medical Ethics Committee of the Republic of Slovenia (reference number: KME 127/10/13). Written informed consent was obtained from all of the donors included in this study.

\section{Consent to Participate}

Written informed consent was obtained from all of the patients included in this study.

\section{Consent for Publication}

Written informed consent for publication was obtained from all of the patients included in this study.

\section{Availability of Data and Material}

All data generated and/or analysed during this study are included in this published article.

\section{Code Availability}

Not applicable.

\section{Authors' Contribution}

Both authors were involved in the design of the study and collection of the data. MŠ was responsible for the statistical analysis. Both authors wrote the manuscript and were involved in the final approval of the manuscript.

\section{Acknowledgment}

We are thankful to the staff of the Laboratory for Haemostasis and Atherothrombosis at the Clinical Department of Angiology, University Clinical Centre Ljubljana for serum biomarker measurements and technical assistance. The authors acknowledge Chris Berrie for scientific English editing of the manuscript.

\section{References}

1. Fallen EL (2003) Coronary artery disease, inflammation and the ghost of John Hunter. Inflammopharmacology 11: 195-201.

2. Koenig W (2013) High-sensitivity C-reactive protein and atherosclerotic disease: From improved risk prediction to risk-guided therapy. Int J Cardiol 168: 5126-5134.

3. Sugiura T, Yoshikawa D, Ishii H, Soichiro Kumagai, Yosuke Inoue, et al. (2014) Relation of omega-3 fatty acid and C-reactive protein to peripheral artery disease in patients with coronary artery disease. Heart Vessels 29: 449-455.

4. Eisenhardt SU, Habersberger J, Oliva K, Mustafa Ayhan, Kevin J Woollard, et al. (2011) A proteomic analysis of C-reactive protein stimulated THP1 monocytes. Proteome Sci 9.

5. Murphy TM, Baum LL, Beaman KD (1991) Extrahepatic transcription of human C-reactive protein. J Exp Med 173: 495-498.

6. Pepys MB, Hirschfield GM (2003) C-reactive protein: a critical update. J Clin Invest 111: 1805-1812.
7. Mizia-Stec K, Gasior Z, Zahorska-Markiewicz B, Michał Holecki, Paweł Kumor (2006) Inflammatory markers in a 2-year follow-up of coronary artery disease. Hear Vessel 21: 302-308.

8. Hartman J, Frishman WH (2014) Inflammation and atherosclerosis: A review of the role of interleukin-6 in the development of atherosclerosis and the potential for targeted drug therapy. Cardiol Rev 22:147-151.

9. Walsh BW, Cox DA, Sashegyi A, RA Dean, RP Tracy, et al. (2001) Role of tumor necrosis factor-alpha and interleukin-6 in the effects of hormone replacement therapy and raloxifene on C-reactive protein in postmenopausal women. Am J Cardiol 88: 825-828.

10. Andersson J, Libby P, Hansson GK (2010) Adaptive immunity and atherosclerosis. Clin Immunol 134: 33-46.

11. Cavusoglu E, Marmur JD, Hojjati MR, Vineet Chopra, Mitul Butala, et al. (2011) Plasma interleukin-10 levels and adverse outcomes in acute coronary syndrome. Am J Med 124: 724-730.

12. Luc G, Arveiler D, Evans A, Philippe Amouyel, Jean Ferrieres, et al. (2003) Circulating soluble adhesion molecules ICAM- 1 and VCAM- 1 and incident coronary heart disease: The PRIME Study. In: Atherosclerosis pp. 169-176.

13. Siddiqui MAA, Keam SJ (2006) Spotlight on ranolazine in chronic stable angina pectoris. Am J Cardiovasc Drugs 6: 357-359.

14. Lanza GA, Crea F (2016) Overview of management of myocardial ischemia: a mechanistic-based approach. Cardiovasc Drugs Ther 30: 341-349.

15. Deshmukh SH, Patel SR, Pinassi E, Eileen V Hermance, Michael N Infantino, et al. (2009) Ranolazine improves endothelial function in patients with stable coronary artery disease. Coron Artery Dis 20: 343347.

16. Lin X, Ma A, Zhang W, Qun Lu, Chaofeng Sun, et al. (2013) Cardioprotective effects of atorvastatin plus trimetazidine in percutaneous coronary intervention. Pakistan J Med Sci 29.

17. Kuralay F, Altekin E, Yazlar AS, Banu Onvural, Ozhan Goldeli (2006) Suppression of angioplasty-related inflammation by pre- procedural treatment with trimetazidine. Tohoku J Exp Med 208: 203-212.

18. Knuuti J, Wijns W, Achenbach S, Davide Capodanno, Emanuele Barbato, et al. (2020) 2019 ESC guidelines for the diagnosis and management of chronic coronary syndromes. Eur Heart J 41: 407-477.

19. Erdfelder E, FAul F, Buchner A, Lang AG (2009) Statistical power analyses using $\mathrm{G}^{*}$ Power 3.1: Tests for correlation and regression analyses. Behav Res Methods 41: 1149-1160.

20. Friedewald WT, Levy RI, Fredrickson DS (1972) Estimation of the concentration of low-density lipoprotein cholesterol in plasma, without use of the preparative ultracentrifuge. Clin Chem 18: 499-502.

21. Ridker PM, Rifai N, Pfeifer MA, F Sacks, E Braunwald (1999) Long-term effects of pravastatin on plasma concentration of C- reactive protein. Circulation 100: 230-235.

22. Strandberg TE, Vanhanen H, Tikkanen MJ (2000) Associations between change in C-reactive protein and serum lipids during statin treatment. Ann Med 32: 579-583.

23. Kinlay S, Schwartz GG, Olsson AG, Nader Rifai, Sally J Leslie, et al. (2003) High-dose atorvastatin enhances the decline in inflammatory markers in patients with acute coronary syndromes in the MIRACL study. Circulation 108: 1560-1566.

24. Šebeštjen M, Keber I, Žegura B, Sasa Simcic, Mojca Bozic, et al. (2004) Statin and fibrate treatment of combined hyperlipidemia: The effects on some novel risk factors. Thromb Haemost 92: 1129-1135.

25. Zakynthinos E, Pappa N (2009) Inflammatory biomarkers in coronary artery disease. J Cardiol 53: 317-333. 
26. Wang XH, Liu SQ, Wang YL, Jin Y (2014) Correlation of serum highsensitivity C-reactive protein and interleukin- 6 in patients with acute coronary syndrome. Genet Mol Res 13: 4260-4266.

27. Tsikouris JP, Suarez JA, Simoni JS, Ziska M, Meyerrose GE (2004) Exploring the effects of ACE inhibitor tissue penetration on vascular inflammation following acute myocardial infarction. Coron Artery Dis 15: 211-217.

28. Schieffer B, Bünte C, Witte J, Kirsten Hoeper, Rainer H Böger, et al. (2004) Comparative effects of AT1-antagonism and angiotensin-converting enzyme inhibition on markers of inflammation and platelet aggregation in patients with coronary artery disease. J Am Coll Cardiol 44: 362-368.

29. Nissen SE, Tuzcu EM, Schoenhagen P, Tim Crowe, BS, William J Sasiela, et al. (2005) Statin therapy, LDL cholesterol, C-reactive protein, and coronary artery disease. N Engl J Med 352: 29-38.

30. Ikonomidis I, Andreotti F, Economou E, C Stefanadis, P Toutouzas, et al. (1999) Increased proinflammatory cytokines in patients with chronic stable angina and their reduction by aspirin. Circulation 100: 793-798.

31. Pudil R, Pidrman V, Krejsek J, J Gregor, M Tichý, et al. (2001) The effect of trimetazidine on C-reactive protein, cytokines and adhesion molecules in the course of acute myocardial infarction. Acta Medica (Hradec Kralove) 44(4): 135-140

32. Szkodzinski J, Danikiewicz A, Hudzik B, Ilona Korzonek-Szlacheta, Mariusz Gąsior, et al. (2015) Effect of trimetazidine on serum interleukin- 6 and C- reactive protein concentrations in patients with stable coronary artery disease. J Biol Regul Homeost Agents 95(6):759762.

33. Martins GF, Filho AG de S, Santos JB de F, Claudio Roberto Cavalcanti Assunção, Francisca Bottino Vieira, et al. (2012) Trimetazidine and inflammatory response in coronary artery bypass grafting. Arq Bras Cardiol 99: 688-696.

34. Wan S, De Smet JM, Barvais L, M Goldstein, JL Vincent, et al. (1996) Myocardium is a major source of proinflammatory cytokines in patients undergoing cardiopulmonary bypass. J Thorac Cardiovasc Surg 112: 806-811.

ISSN: 2574-1241

DOI: 10.26717/BJSTR.2021.34.005597

Miran Šebeštjen. Biomed J Sci \& Tech Res

(C) This work is licensed under Creative Commons Attribution 4.0 License

Submission Link: https://biomedres.us/submit-manuscript.php
35. Di Napoli P, Taccardi AA, Barsotti A (2005) Long term cardioprotective action of trimetazidine and potential effect on the inflammatory process in patients with ischaemic dilated cardiomyopathy. Heart 91: 161-165.

36. Caligiuri G, Rudling M, Ollivier V, Marie-Paule Jacob, Jean-Baptiste Michel, et al. (2003) Interleukin- 10 deficiency increases atherosclerosis, thrombosis, and low-density lipoproteins in apolipoprotein E knockout mice. Mol Med 9: 10-17.

37. Heeschen C, Dimmeler S, Hamm CW, Stephan Fichtlscherer, Eric Boersma, et al. (2003) Serum level of the antiinflammatory cytokine interleukin-10 is an important prognostic determinant in patients with acute coronary syndromes. Circulation 107: 2109-2114.

38. Lakoski SG, Liu Y, Brosnihan KB, Herrington DM (2008) Interleukin-10 concentration and coronary heart disease (CHD) event risk in the estrogen replacement and atherosclerosis (ERA) study. Atherosclerosis 197: 443-447.

39. Welsh P, Murray HM, Ford I, Anton J M de Craen, J Wouter Jukema, et al. (2011) Circulating interleukin-10 and risk of cardiovascular events: A prospective study in the elderly at risk. Arterioscler Thromb Vasc Biol 31: 2338-2344.

40. Wanidworanun C, Strober W (1993) Predominant role of tumor necrosis factor-alpha in human monocyte IL-10 synthesis. J Immunol 151: 68536861.

41. Stumhofer JS, Silver JS, Laurence A, Paige M Porrett, Tajie H Harris, et al. (2007) Interleukins 27 and 6 induce STAT3-mediated T cell production of interleukin 10. Nat Immunol 8: 1363-1371.

42. Goldwater D, Karlamangla A, Merkin SS, Karol Watson, Teresa Seeman (2019) Interleukin-10 as a predictor of major adverse cardiovascular events in a racially and ethnically diverse population: Multi-Ethnic Study of Atherosclerosis. Ann Epidemiol 30: 9-14. e1.

43. Inoue T, Komoda H, Nonaka M, Miho Kameda, Toshihiko Uchida, et al. (2008) Interleukin-8 as an independent predictor of long-term clinical outcome in patients with coronary artery disease. Int J Cardiol 124: 319325 .

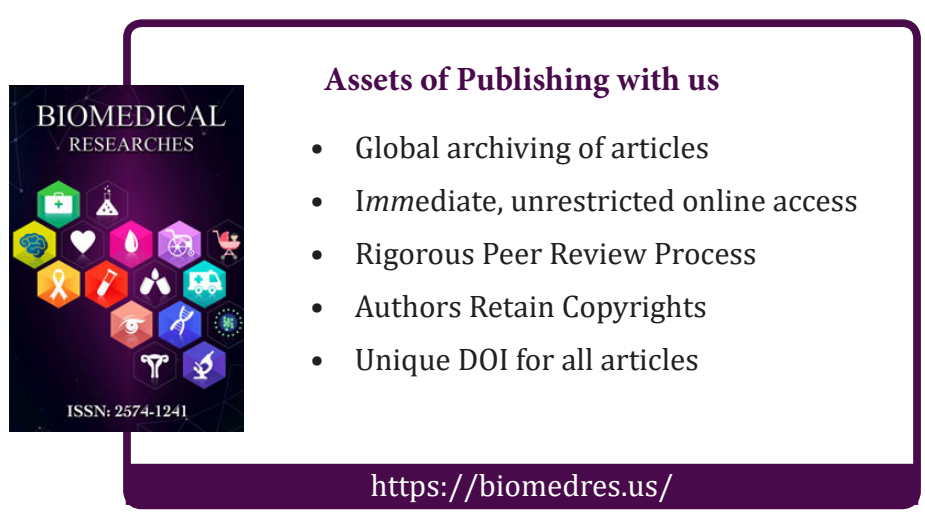

